\title{
The trapped region
}

\author{
Lars Andersson \\ Albert Einstein Institute, Am Mühlenberg 1, D-14467 Golm, Germany
}

\begin{abstract}
I will discuss some recent results on marginally outer trapped surfaces, apparent horizons and the trapped region. A couple of applications of the results developed for marginally outer trapped surfaces to coalescence of black holes and to the characterization of the trapped region are given.
\end{abstract}

Keywords: black holes, apparent horizons, marginally trapped surfaces

PACS: 02.40-k,04.20-q,04.70-Bw

\section{INTRODUCTION}

Marginally outer trapped surfaces are natural candidates for quasi-local black hole boundaries in general relativity. They are analogues of minimal surfaces in Riemannian geometry, and in particular there is a notion of stability for marginally outer trapped surfaces, closely related to stability for minimal surfaces, which allows one to prove curvature bounds analogous to those which are known for minimal surfaces.

It is natural to consider outermost marginally outer trapped surfaces, which enclose every weakly outer trapped surface. For these, we have area bounds, as well as a replacement for the strong maximum principle, which sheds light on the process of black hole coalescence.

\subsection{Notation}

Let $(M, g, K)$ be a Cauchy hypersurface in a $3+1$ dimensional Lorentzian spacetime $\mathscr{M}$. Let $\Sigma$ be a spacelike surface in $M$ with null normals $\ell^{ \pm}$, see figure 1 . We set

$$
\begin{aligned}
A & =\langle\nabla . v, \cdot\rangle, \quad K^{\Sigma}=\left.K\right|_{T \Sigma \times T \Sigma}, \\
\chi^{ \pm} & =K^{\Sigma} \pm A,
\end{aligned}
$$

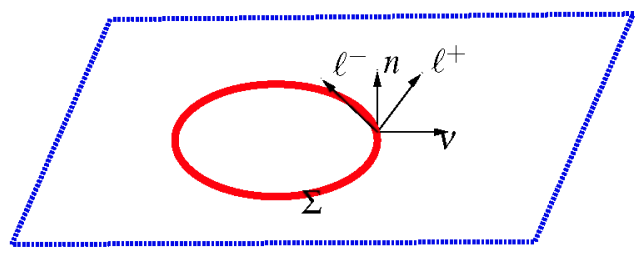

FIGURE 1. 
Then $A$ is the second fundamental form of $\Sigma$ in $M, K^{\Sigma}$ is the restriction of $K$ to $\Sigma$, and $\chi^{ \pm}$are the null second fundamental forms associated to $\ell^{ \pm}$. Taking traces yields

$$
H=\Sigma_{\operatorname{tr} A,} \quad P=\Sigma_{\operatorname{tr} K^{\Sigma}}=\operatorname{tr} K-K(v, v), \quad \theta^{ \pm}=\Sigma_{\operatorname{tr}} \chi^{ \pm}=P \pm H .
$$

Then $H$ is the mean curvature of $\Sigma$ in $M, P$ is the trace of $K^{\Sigma}$ on $\Sigma$ and $\theta^{ \pm}$are the null expansions of $\Sigma$. We declare $\ell^{+}$to be the outer null normal.

Definition $1 \Sigma$ is a marginally outer trapped surface (MOTS) if $\theta^{+}=0$.

Recall that $\theta^{+}$is the logarithmic variation of area along $\ell^{+}$,

$$
\theta^{+}=\delta_{\ell^{+}} \mu_{\Sigma} / \mu_{\Sigma}
$$

If $\Sigma$ is a MOTS then outgoing null rays are marginally collapsing. We call $\Sigma$ (weakly) outer trapped if $\left(\theta^{+} \leq 0\right) \theta^{+}<0$. The null energy condition (NEC) holds if $G(v, v) \geq 0$ for any null vector $v$, where $G=\operatorname{Ric}-\frac{1}{2} \operatorname{Sc} g$ is the Einstein tensor.

The usual definition of trapped surface is $\theta^{+}<0, \theta^{-}<0$. By the singularity theorems of Hawking and Penrose, a maximal globally hyperbolic spacetime satisfying suitable energy conditions, eg., NEC, and which contains a trapped surface, is causally incomplete. We note that also the presence of an outer trapped surface implies incompletness. In particular, if NEC holds in $\mathscr{M}$ and $\mathscr{M}$ contains a Cauchy surface $M$ with an outer trapped surface which separates and has noncompact exterior, then $\mathscr{M}$ is null geodesically incomplete, $\mathrm{cf}$. $[1,2]$. Thus MOTSs may be viewed as black hole boundaries.

The null expansion $\theta^{+}=P+H$ is elliptic when viewed as a functional of $\Sigma \subset M$, since, as is well known, the mean curvature $H$ has this property, and $P$ may be viewed as a lower order term. For variations along $\ell^{+}$, we have

$$
\delta_{f \ell^{+}} \theta^{+}=-W f=-\left(\left|\chi^{+}\right|^{2}+G\left(\ell^{+}, \ell^{+}\right)\right) f
$$

so $|\Sigma|$ is not an elliptic functional with respect to null variations. However, variations within $M$, of the form $\delta_{f v} \theta^{+}$define an elliptic operator, see section 1.2 below.

It is well known that if there are surfaces $\Sigma_{ \pm}$with $H\left[\Sigma_{+}\right]>0$ and $H\left[\Sigma_{-}\right]<0$, which form barriers for the problem of minimizing area, then there is a minimal $(H=0)$ surface between them. Suppose we have an analogue for MOTSs of existence in the presence of barriers, in this case surfaces $\Sigma_{ \pm}$with the inner barrier satisfying $\theta^{+}\left[\Sigma_{-}\right]<0$, while the outer barrier satisfies $\theta^{+}\left[\Sigma_{+}\right]>0$. Then, in view of (1), MOTSs should persist if NEC holds. This can in fact be proved using the results of [3], given an outer barrier, cf. [2], see section 2.2. In particular, if $(M, g, K)$ is an asymptotically flat initial data set, then there is an outer barrier in $M$. We shall, throughout the rest of this note, assume the presence of an outer barrier.

Due to the persistence of MOTSs, we expect that MOTSs are generically in a marginally outer trapped tube (MOTT), i.e. a hypersurface of $\mathscr{H}$, foliated by MOTSs. This has been proved for outermost MOTSs, modulo a genericity condition, cf. theorem 1 and [2]. In this case, the MOTT is weakly spacelike if NEC holds. If in addition $\theta^{-}<0$ on the MOTS, the MOTT is a dynamical horizon, cf. $[4,5]$. 


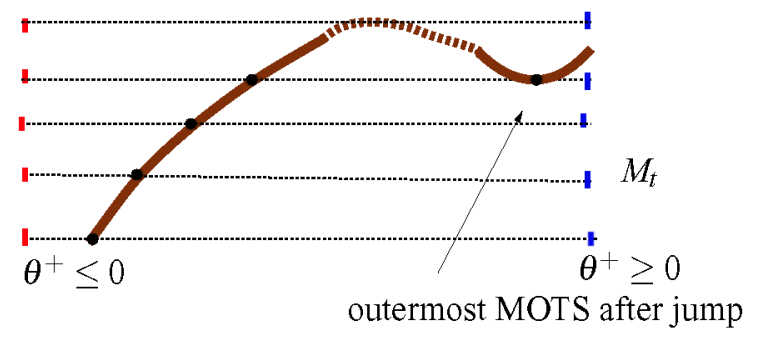

FIGURE 2.

\subsection{The stability operator}

Let $L f=\delta_{f v} \theta^{+}$. Then

$$
L f=-\Delta f+2 S(\nabla f)+f\left[\operatorname{div} S-|S|^{2}-\frac{1}{2}\left|\chi^{+}\right|^{2}+\frac{1}{2} \Sigma \mathrm{Sc}+(\mu-J(v))\right]
$$

where $S(X)=K(X, v)$. The operator $L$ is the analogue of the minimal surface stability operator. We note the following facts which hold for $L$. The operator $L$ is 2 :nd order elliptic and non-self adjoint in general. There is a unique principal eigenvalue $\lambda \in \mathbb{R}$, with positive eigenfunction $\phi$. If $\Sigma$ a locally outermost MOTS then $\lambda \geq 0$. Further, if $\lambda \geq 0$ then there is $f \geq 0$ such that $L f \geq 0$, i.e., if $\lambda \geq 0$ the maximum principle holds.

Definition $2 \Sigma$ is stable if $\lambda \geq 0$.

In particular, if $\Sigma$ locally outermost then $\Sigma$ is stable.

\subsection{Local existence of horizons}

The proof of the following theorem makes use of the definition of $L$, the implicit function theorem, as well as the above mentioned version of the maximum principle. The following theorem was proved in $[6,2]$.

Theorem 1 Suppose $\Sigma$ is stable $(\lambda \geq 0)$. If $\lambda=0$, assume in addition ${ }^{1} W$ is not identically zero. Then $\exists$ a MOTT $\mathbf{H}$ containing $\Sigma$. $\mathbf{H}$ is weakly spacelike if NEC holds.

Theorem 1 is a local result. In general, the outermost MOTS can jump. This may happen for example through the formation of a new MOTS outside the existing ones, a process that can be caused by the coalescence of black holes, cf. section 3.1. As a MOTS is created, the MOTT bifurcates in general, see [2], see also figure 2.

\footnotetext{
${ }^{1}$ The condition that $W$ is not identically zero may be viewed as a genericity condition
} 


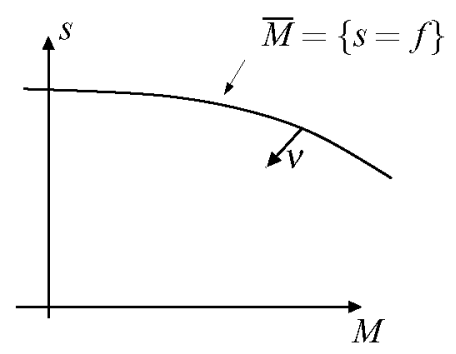

FIGURE 3.

\subsection{Curvature bounds}

Theorem 2 ([7]) Let $\Sigma$ be a stable MOTS. Then

$$
|A| \leq C\left(|\operatorname{Riem}|_{C^{0}},|K|_{C^{1}}, \operatorname{inj}(M)\right)
$$

The proof of theorem 2 applies several techniques used in [8], including the Simons identity, a Kato inequality, and the Hoffmann-Spruck Sobolev inequality. The Moser iteration used in [8] to achieve the $L^{\infty}$ estimate for $|A|$ is replaced by a Stampacchia iteration. Further, the symmetrized stability estimate of [9] is used. By applying the local area bound of Pogorelov, it is possible to avoid a dependence on the area $|\Sigma|$ in the curvature estimate. Due to the use of this result, which in turn relies upon the GaussBonnet theorem, the above form of the curvature estimate applies only to the case of a 2-dimensional surface in a $3+1$ dimensional spacetime.

\section{EXISTENCE OF MOTS}

\subsection{Jang's equation}

Consider $\mathbb{R} \times M$, with metric $d s^{2}+g$, see figure 3. Define $\bar{K}$ by pullback of $K$. Let $\bar{M}$ be the graph of $f$. On $\bar{M}$ we have induced mean curvature $\mathscr{H}$ and $\mathscr{P}=\operatorname{tr}_{\bar{M}} \bar{K}$. Jang's equation is

$$
\mathscr{J}[f]:=\mathscr{H}-\mathscr{P}=0
$$

This is the analogue of the equation $\theta^{+}=0$

By translation invariance, $J[f]=J[f+t]$, we have that the stability operator for $\bar{M}$ has $L \phi=0$, with $\phi=\left\langle v, e_{4}\right\rangle$. Here $L$ is the analogue of the minimal surface stability operator for $\bar{M}$. By the work in [10], we have local curvature bounds for $\bar{M}$, which yields compactness. This allows one to prove existence of solutions to Jang's equation using a capillarity deformation together with Leray-Schauder theory. One considers the deformed equation

$$
\mathscr{H}-\sigma \mathscr{P}=\tau f_{\sigma, \tau}
$$

As $\sigma$ goes from 0 to 1 , we have a limit $f_{\tau}$. Letting $\tau \searrow 0$, we have by compactness, convergence of a subsequence of $f_{\tau}$ to a solution $f$ of Jang's equation. The solution 


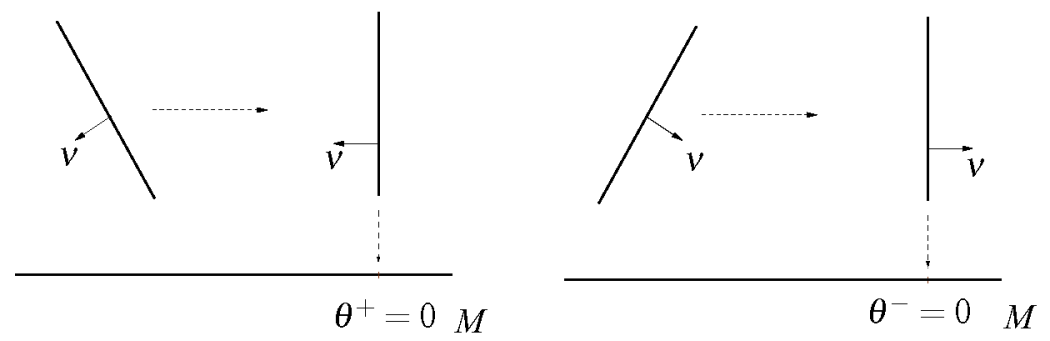

FIGURE 4.

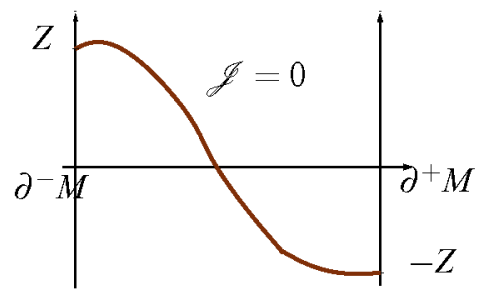

FIGURE 5.

has blowups in general. As observed in [10], blowups project to MOTSs, cf. figure 4. Therefore Jang's equation can be used to prove existence of MOTSs. It was proved in [3] that the blowup surfaces are stable MOTSs.

Theorem 3 ([11, 3]) Suppose $M$ is compact with barrier boundaries $\partial^{ \pm} M$, such that

$$
\theta^{+}\left[\partial^{-} M\right]<0, \quad \theta^{+}\left[\partial^{+} M\right]>0
$$

Then $M$ contains a MOTS $\Sigma$.

Theorem 3 provides the analogue of the barrier argument for existence of minimal surfaces. The proof considers a sequence of Dirichlet problems for Jang's equation, which forces a blowup solution. We solve

$$
\mathscr{J}[f]=0,\left.\quad f\right|_{\partial^{ \pm} M}=\mp Z
$$

see figure 5 . If we let $Z \rightarrow \infty$, then the solution converges to solution with blowups.

In order to prove boundary gradient estimates necessary to apply Leray-Schauder theory, one makes use of a deformation of the Cauchy surface, see figure 6 , to get $H>0$ at $\partial M$. The limiting solution must blow up somewhere, which implies the existence of a MOTS. We have a foliation by barriers near $\partial M$. Using this fact, and the maximum principle, one can show the MOTSs constructed are in the undeformed region of $M$.

By deforming the data inside $\partial^{-} M$, we can allow $\theta^{+}\left[\partial^{-} M\right] \leq 0$. We remark that $[12]$ has studied the Plateau problem for MOTSs using Perron's method. 


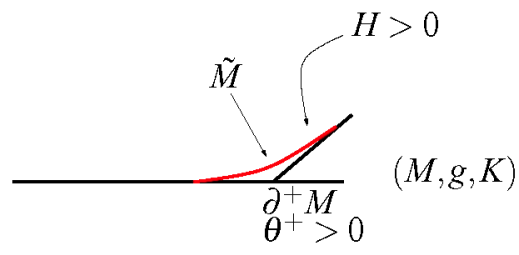

FIGURE 6.

\subsection{Application: Persistence of MOTSs}

By Rauchaudhuri, $\delta_{\ell^{+}} \theta^{+}=-W \leq 0$ if NEC holds.

Theorem 4 ([2]) Let $\mathscr{M}$ be a spacetime which satisfies NEC. Let $\left\{M_{t}\right\}$, be a Cauchy foliation of $\mathscr{M}$, and assume we have outer barriers.

If $M_{0}$ contains a MOTS, then each $M_{t}, t \geq 0$ contains a MOTS.

Based on theorem 4, it seems natural to view the collection of outermost MOTS in $M_{t}$ as the black hole boundary in $\mathscr{M}$. If it is smooth, this collection is a MOTT. For further regularity and continuation results for MOTTs, cf. [2].

\section{AREA BOUND}

Theorem 5 ([3]) Suppose $M$ has outer barrier. There is a constant $C=C\left(\mid\right.$ Riem $\left.\right|_{C^{0}}$, $\left.|K|_{C^{1}}, \operatorname{inj}(M), \operatorname{Vol}(M)\right)$ such that for a bounding MOTS $\Sigma$ in $M$, either

$$
|\Sigma| \leq C
$$

or there is a MOTS $\Sigma^{\prime}$ outside $\Sigma$.

The idea of proof of theorem 5 is the following. If $|\Sigma|$ is very large, then due to curvature bounds and the bounded $\operatorname{Vol}(M), \Sigma$ must nearly meet itself from the outside, which implies that the outer injectivity radius $i^{+}(\Sigma)$ must be small. In this situation we can use surgery and heat flow to show the existence of a MOTS outside $\Sigma$. In a location where $\Sigma$ nearly meets itself on the outside, we glue in a neck with $\theta^{+}<0$, see figure 7 . The resulting surface is then deformed using the $\theta^{+}$heat flow

$$
\dot{x}=-\theta^{+} v,
$$

this gives a family $\Sigma_{s}, s \geq 0$. The maximum principle can be used to show that for $s>0$, $\Sigma_{s}$ is outside $\Sigma$, with $\theta^{+}\left[\Sigma_{S}\right]<0$. Thus, $\Sigma_{s}$ is an inner barrier, which means that we can apply the existence result theorem 3. It follows there is a MOTS $\Sigma_{\text {new }}$ outside $\Sigma$. Each time the above argument is applied it uses at least $\delta_{\mathrm{Vol}}$ of the volume outside $\Sigma$, and hence after finitely many steps, one has a $\Sigma_{0}$ outside $\Sigma$ with outer injectivity radius $i^{+}\left(\Sigma_{0}\right)>\delta_{*}$. The surface $\Sigma_{0}$ has the claimed area bound. To estimate the area, we use the estimates on curvature and $i^{+}$to estimate the volume of a tube around $\Sigma_{0}$ from below, using the divergence theorem, in terms of $\left|\Sigma_{0}\right|$. This tube must have volume bounded by $\operatorname{Vol}(M)$, 

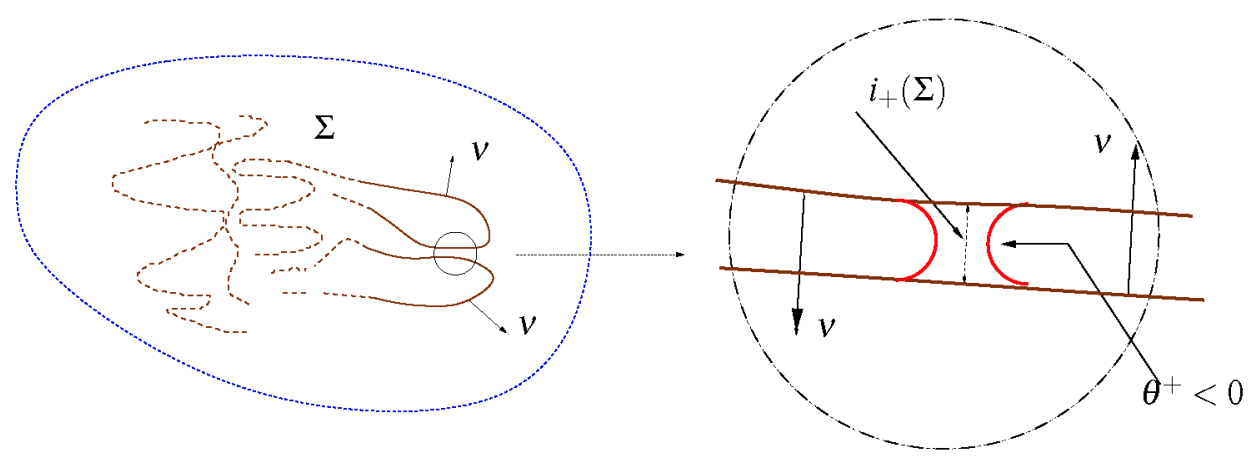

FIGURE 7. Gluing in a neck with $\theta^{+}<0$.

which leads to an estimate for $\left|\Sigma_{0}\right|$. This area bound, together with the curvature bound from Theorem 2, gives compactness for the family of outermost MOTSs in a sequence of Cauchy data sets with suitable uniformity properties, $\mathrm{cf}$. [3].

\subsection{Application: Coalescence of black holes}

It is a direct consequence of the gluing and heat flow construction used in the proof of the area bound, that if $\Sigma_{1}, \Sigma_{2}$ are locally outermost MOTSs which are sufficiently close, then there is a MOTS $\Sigma$ surrounding them. This may be interpreted as stating that black holes must coalesce once they are sufficiently close. The phenomenon described here is seen in numerical simulations. As the MOTS $\Sigma$ is formed in an evolution, it has principal eigenvalue $\lambda=0$ and generically there is a MOTT which bifurcates into existence when $\Sigma$ is formed. See [2] for details.

Remark 3.1 The above result gives a "maximum principle for MOTS". Note that the usual maximum principle does not apply for MOTSs which meet on the outside.

\section{THE TRAPPED REGION}

Definition 3 Let $(M, g, K)$ be an $A F$ data set. The trapped region is

$$
\mathbb{T}=\cup_{\Omega \subset M}\{\partial \Omega \quad \text { is weakly outer trapped }\}
$$

Theorem 6 ([3]) If $\exists \Omega \subset M$, with $\partial \Omega$ weakly outer trapped, then $\mathbb{T}$ has smooth boundary $\partial \mathbb{T}$, with $\theta^{+}[\partial \mathbb{T}]=0$. In particular, $\partial \mathbb{T}$ is the unique outermost MOTS in $M$.

For the proof, replace $\mathbb{T}$ by

$$
\mathscr{T}=\cup_{\Omega}\left\{\theta^{+}[\partial \Omega] \leq 0, \quad \text { and } i^{+}(\partial \Omega) \geq \delta_{*}\right\}
$$

For the collection of subsets defining $\mathscr{T}$ we have compactness by the area bound for surfaces with $i^{+}$bounded from below. This, together with a gluing construction to 


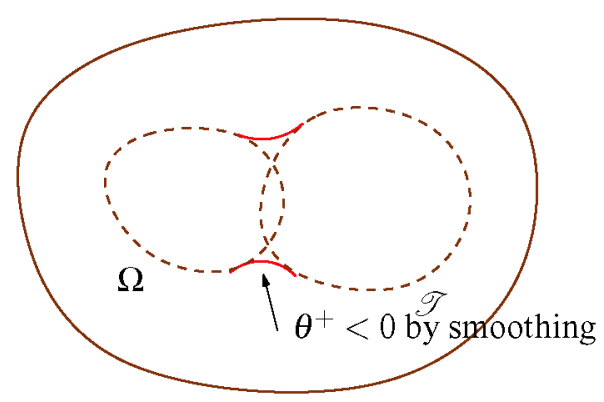

FIGURE 8.

smooth corners gives that $\partial \mathscr{T}=\Sigma$ is a MOTS. To complete the proof we have to show that $\mathbb{T} \subset \mathscr{T}$. To see this, suppose there is a weakly outer trapped surface $\Omega \nsubseteq \mathscr{T}$. We can argue that this means $\Omega \cap \mathscr{T} \neq 0$. Smoothing gives a barrier, see figure 8 and hence there is a MOTS outside. This can be taken to be in $\mathscr{T}$. Hence $\mathbb{T}=\mathscr{T}$, which completes the proof.

Remark 4.1 By [13], the outermost MOTS is a union of finitely many $S^{2}$, assuming NEC.

\section{CONCLUDING REMARKS}

Bray and Khuri have proposed generalized apparent horizons (GAH), satisfying the condition $H=|P|$ as well as a generalized Jang's equation motivated by the GAH condition, as part of an approach to the general Penrose Inequality. [14] proved existence of outermost GAH. These are area outer minimizing. However, it is not clear how they are related to black holes. Large families of GAH conditions can be treated using the techniques discussed here.

Global properties of MOTTs may be relevant for understanding the strong field Cauchy problem for the Einstein equations.

The known conditions for existence of MOTSs in a Cauchy data set $[15,16,17]$ involve nonvacuum data. A better understanding of conditions for the existence of MOTSs in vacuum, due to concentration of curvature in terms of, say, curvature radii, conformal spectral gap, etc. is needed.

\section{Acknowledgements}

I thank the Mittag-Leffler-Institute, Djursholm, Sweden for hospitality and support. This work was supported in part by the NSF, under contract no. DMS 0407732 and DMS 0707306 with the University of Miami. 


\section{REFERENCES}

1. D. Gannon, General Relativity and Gravitation 7, 219-232 (1976).

2. L. Andersson, M. Mars, J. Metzger, and W. Simon (2008), 0811.4721.

3. L. Andersson, and J. Metzger (2007), 0708.4252.

4. A. Ashtekar, and B. Krishnan, Phys. Rev. Lett. 89, 261101,4 (2002), ISSN 0031-9007.

5. A. Ashtekar, and G. J. Galloway, Adv. Theor Math. Phys. 9, 1-30 (2005), ISSN 1095-0761.

6. L. Andersson, M. Mars, and W. Simon, Phys. Rev. Lett. 95, 111102 (2005), gr-qc/ 0506013.

7. L. Andersson, and J. Metzger (2005), gr-qc/0512106.

8. R. Schoen, L. Simon, and S. T. Yau, Acta Math. 134, 275-288 (1975), ISSN 0001-5962.

9. G. J. Galloway, and R. Schoen, Comm. Math. Phys. 266, 571-576 (2006), ISSN 0010-3616.

10. R. Schoen, and S. T. Yau, Comm. Math. Phys. 79, 231-260 (1981), ISSN 0010-3616.

11. R. Schoen (2004).

12. M. Eichmair, The Plateau problem for apparent horizons (2007), arXiv.org:0711.4139.

13. G. J. Galloway, Comm. Anal. Geom. 16, 217-229 (2008), ISSN 1019-8385.

14. M. Eichmair, Existence, regularity, and properties of generalized apparent horizons (2008), arXiv.org:0805.4454.

15. R. Schoen, and S. T. Yau, Comm. Math. Phys. 90, 575-579 (1983), ISSN 0010-3616.

16. S. T. Yau, Adv. Theor: Math. Phys. 5, 755-767 (2001), ISSN 1095-0761.

17. G. J. Galloway, and N. O'Murchadha, Class. Quant. Grav. 25, 105009 (2008), 0802.3247. 